\title{
Urban complexity, scale hierarchy, energy efficiency and economic value creation
}

\author{
S. Salat \& L. Bourdic \\ Urban Morphology Lab, CSTB, Paris, France
}

\begin{abstract}
Several authors, such as Batty or Frankhauser have already analysed cities as fractal structures. Putting in perspective historical cities with modernist cities, others such as Alexander or Salingaros have stressed the loss of urban complexity over time. This paper aims at going a step further and investigates energy and economic aspects of cities through the prism of complexity theory. Fostering a more systematic use of fractal approaches, the authors highlight the crucial role that urban complexity plays in urban sustainability: only scale hierarchic urban structures will succeed in optimising the use of scarce resources allocated, improving at the same time energy efficiency and economic value creation.
\end{abstract}

Keywords: power laws, fractals, urban efficiency, sustainable cities.

\section{Introduction}

Contrary to Vienna, Barcelona or Kyoto, Paris and Tokyo have grown without a proper master plan. The plans of Paris and Tokyo though are never incoherent: on every scale, patterns prove to be strikingly stable, as for example the size and structure of the urban grid. In both cases, the layout is immediately picked up by a remarkably dense geometry. In addition, in both cases, the city has adapted differently to its culture and its time. Faced with these cities without plan but which metric and topologic structures are extremely stable, two questions might be explored. The first one boils down to asking whether inventing the city, instead of investing in isolated projects, is not about defining rules of assemblage and coexistence of elements. Modernism has impoverished this complexity and often reduced the city to isolated objects. The second question that is the key issue of this paper is about understanding urban complexity and defining the 
thresholds that make a city be a "living city" [1]. But in the current context of climate change and resource scarcity, it is above all about making a city be a resilient and efficient city, in its wider meaning.

The issue of urban complexity has already been tackled from different angles by several authors. In this paper though, authors choose to investigate this issue through a prism that has been first introduced by Benoit Mandelbrot [2]: fractal geometry. Analyzing cities as fractal structures is not new and the contribution of authors like Batty and Longley [3] or Frankhauser [4] is fundamental. But in this paper authors aim at pushing further the analysis toward what makes the very structural complexity of urban structures. The approach rests upon looking at the structural mathematical laws that appear between the lines of fractal theory. Building on Pareto's [5], Zipf's [6] and then Mandelbrot's [7] breakthroughs, authors look further in the direction given by Alexander [8] and Salingaros [1], they investigate in a quantitative way but in a wider sense the potential of scale hierarchy and power laws to make cities structurally efficient, resilient and living.

"Few if any economists seem to have realized the possibilities that such invariants hold for the future of our science. In particular, nobody seems to have realized that the hunt for, and the interpretation of, invariants of this type might lay the foundations for an entirely novel type of theory." Schumpeter [9], on power laws and Pareto distributions

\section{Fractal cities}

In a nutshell, this paper is about bringing things and scales together. It is first about considering several coexisting approaches and trying to make them converge toward a single, generic and comprehensive framework: fractals, power laws, Zipf's laws, Pareto distributions. It is then about considering all the scales that make the city: from the people to the buildings, blocks, neighbourhoods, districts and eventually cities. These urban scales are never independent one from another. Any comprehensive approach of cities has to rest upon these different scales and yet to accurately consider the relationships between these scales. Rather than digging into details into each field, this paper aims at casting the spotlight on the different approaches - both pragmatic and theoretical -, the urban different scales, highlighting the links and relationships among them, and eventually providing a theoretical generic framework for complex urban analysis.

The interest in rank-size and frequency-size distribution is nothing but new. One of the first one to shed light on these phenomena has been Vilfredo Pareto [5] who proved the distribution of incomes within the Italian society to follow a mathematical relationship of the type $P(x>X)=a X^{m}$. Fifty years later, Zipf [6] found an analogue relationship linking cities size (population) and rank within a system of cities: size $=a \cdot r a n k^{m}$. This empirical law - Zipf's law - is nothing else but an example of a power law function, i.e. a function of the type $\mathrm{Y}=\mathrm{aX} \mathrm{X}^{\mathrm{m}}$. Building on this empirical result, Batty [10] and Pumain [11] have explained how cities growth model can generate power law or lognormal distributions 
when plotting cities' population versus cities' rank. This analysis has been further developed for the 200 tallest buildings in the world [12]. Interestingly, the scaling parameter for cities and buildings is not the same. An interesting question to be asked concerns the role and the influence of the scaling parameter on the overall efficiency of the structure.

According to Gabaix [13], numerous authors since such as Champernowne [14], Simon [15] or Mandelbrot [7] have explored the issue or power laws and tried to give an echo to Schumpeter's vision [9]. Mandelbrot's fractal theory [2] has been one of the greatest contribution in this field by providing a framework for the analysis of complex systems such as cities. Fractal theory reveals a hidden order in the morphology of urban fabrics, uncovering internal spatial structures and grids of ordinary geometry [16]. At first, Mandelbrot built on Hurst's work and applied it to market prices variations [7]. Yet the framework he developed afterwards proved to be an incredibly powerful tool, allowing proving amazing regularities in a wide range of both natural and man-made phenomena still unexpected so far [2]. One of the most known examples of fractals in nature relates to the length of the coast of Britain. The paradox associated with the measurement of this length is that it depends on the scale of measurement: the smaller the increment, the longer the measured length. This issue of scale is omnipresent behind many complex structures - especially cities. One of the most significant contribution to the field has been made by Batty and Longley [3] who have applied and developed fractal methods to apply them to cities. Beyond the rank-size analysis of cities and buildings, Chen [17] also establishes a link between urban growth and diffusion, and a fractal distribution of urban population density. Last but not least, the work carried out by Frankhauser [4] provides innovative insights notably by analyzing the boundaries of urban sprawl using fractal methods.

These approach have cast the spotlight on specific situations where scaling appears within urban phenomena. According to authors' understanding though, these approaches could be significantly enriched by focusing on the intrinsic multi-scale structure of cities. Beyond drawing conclusions on the city scale only, the fractal reasoning should lead to a multi-scale and comprehensive framework, able to skillfully encompass urban complexity. Authors' purpose is to make good use of these approaches keeping in mind the challenges urban world will face in the coming decades: resource scarcity, climate change, demographic growth, socio-economic instability and need for development. Authors aim at identifying how a comprehensive fractal understanding of cities may provide new solutions to these urgent issues. To do so, there is need for developing a more systematic approach of urban complexity. There is a need to push further Batty and others' programmatic work and foster a systematic fractal analysis of urban facts. Making good use of this approach may provide useful tools to develop better - more efficient and resilient - urban forms.

In a recent World Bank report, Suzuki et al. [18] insist on the 6 layers forming the city: Customers, Streets, Parcels, Elevation, Land Use, and Real World. Keeping in mind the need for making cities more sustainable, which layer could be better understood and enriched using a fractal analysis? In other 
words, what are the urban facts and structures that gain in efficiency and resilience when distributed according to power laws? The following sections aim at presenting some benefits that could be expected from a systematic fractal approach concerning urban structures. Considering a given urban fact or structure, is there any underlying power law distribution? Is there any physical reason for the emergence of such a power law? What are the optimal distributions in terms of efficiency, resilience, stability, welfare? And eventually, on which tools could a pragmatic and operational use of these results be made, by the different stakeholders?

\section{Urban networks}

The historical European city displayed different modes of fractal growth. In the $18^{\text {th }}$ century, the fractal structure of the city resulted from fragmentation and juxtaposition, with each period standing as a discrete complete unit, capable of growth but only to create a more extended unit. In Paris, Haussmann's very different method ran against the idea of cities conceived by fragments. Instead of adding them, like in London, Haussmannian urbanism overlaid hierarchical grids, each one of which belonged to a star shaped network. Haussmann entirely reorganized the urban fabric, mainly in the centre, with a new grid of streets, boulevards and avenues. But instead of destroying the historical complexity, Haussmann superimposed a larger scale on the existing fabric, increasing at the same time the overall hierarchy of the city (see Figure 1).

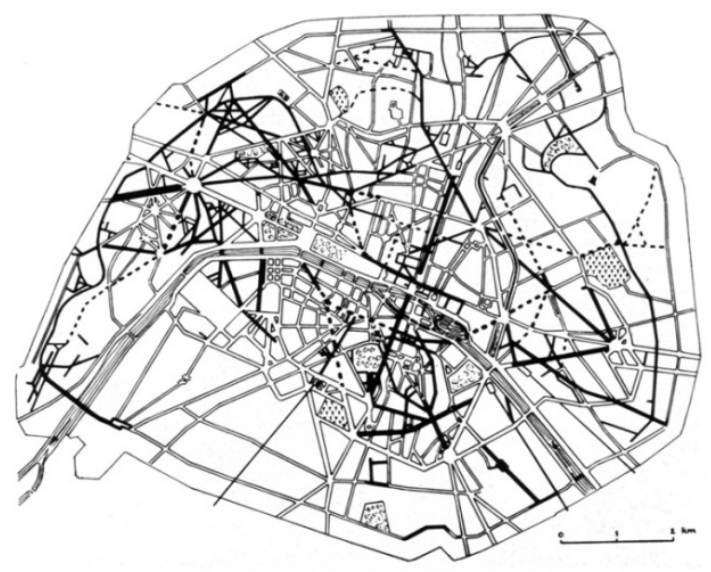

Figure 1: Haussmannian's cuts. Transformation of the street system, implemented or planned during the second empire [16].

A fractal analysis of street networks sheds light on the structure of urban fabrics. Natural structures provide numerous examples of fractal networks that optimize the distribution of fluids: blood, oxygen from the lungs, sap, rivers... Urban street networks can all be analyzed in the light of fractal theory. The first 
step consists in setting up tools and simple methods to analyze the more or less pronounced fractal character of street networks. To do so, authors have developed indicators assessing the deviation of the actual distribution of the network from the theoretical power law. The following analysis (see figure 2) is based on data from the Atelier Parisien d'Urbanisme (APUR). The calculation of the indicator is based on a division into categories of the street network into categories of street width by increments of 8 meters: length of streets from zero to 7 meter wide, from 8 to 15 meter wide, etc. The parameters of the power law are calculated using a bi-logarithmic regression and optimization.

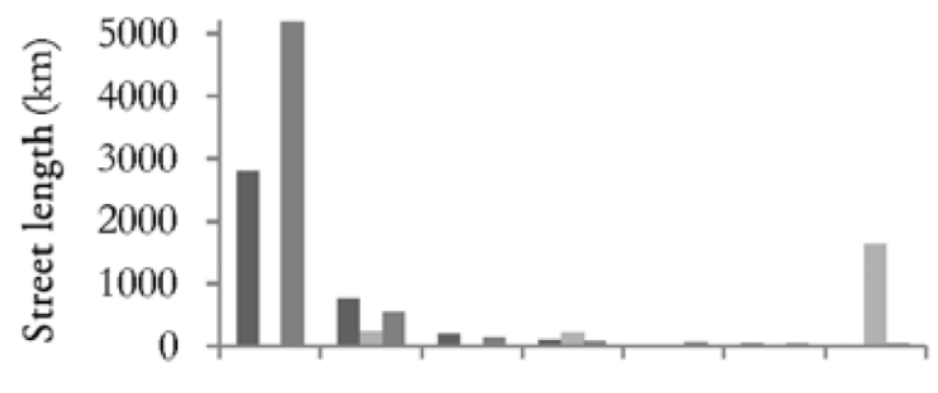

0-7 $\quad 8-15$ 16-23 24-31 32-39 40-47 48-55

\section{Street width (m) \\ - Paris $n$ Le Corbusier $\quad$ Pareto}

Figure 2: Fractal analysis of the Parisian and Corbusean road network. The cumulated street length is plotted vs. the street width $(8 \mathrm{~m}$ width categories).

The same analysis has been carried out on what can be considered as an archetype of the modernist city: the Contemporary City for Three Million Inhabitants by Le Corbusier [19]. The loss of intermediary scales in the Corbusian urban projects is especially noticeable when looking at the structure and hierarchies of road networks (see figure 3). The private car serves as the basic postulate to structuring and sizing the urban network. Le Corbusier does not explicitly refer to the size and distribution of sidewalks and pedestrians streets in his plan. But the preponderance of extremely wide trunk routes and the size of urban grid excludes pedestrian from part of the transportation network. Unlike the Parisian network, The Contemporary City for Three Million Inhabitants project does not respect any scale hierarchy. Worse, for the three unique road sizes, hierarchy is inversed: $240 \mathrm{~km}$ of 10 meter wide private streets, $220 \mathrm{~km}$ of 30 meter wide streets and 1,640 km of 50 meter wide boulevards. The street network width-size distribution is displayed on figure 2 for the Parisian network, the Corbusean one, and the associated theoretical power law distribution. The scale hierarchy of the Parisian network is close to the power law distribution. The indicator of deviation from the power law distribution is 
extremely low (0.17). This result is a quantitative confirmation of the fractal structure and the scale hierarchy of the Parisian network. On the contrary, the hierarchy inversion is immediately discernible when looking at the deviation indicator between Le Corbusier's network distribution and the power law (taken identical to the one for Paris): it is equal to 509. The extremely high value of the deviation indicator demonstrates that the organization of scales in the Corbusean network is reversed.

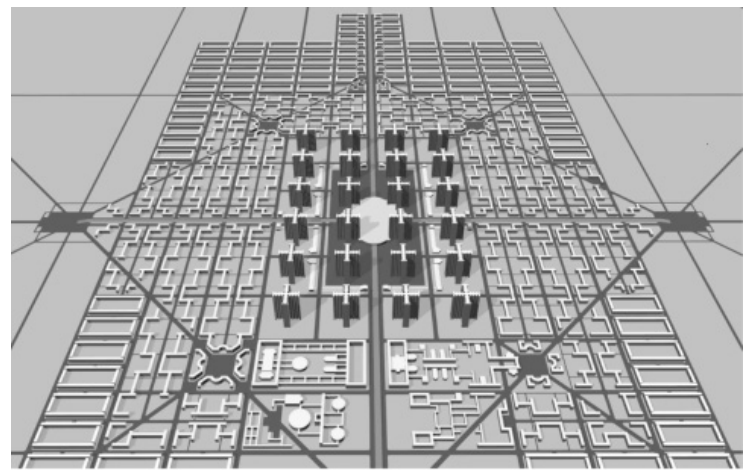

Figure 3: $\quad$ Le Corbusier [16].

There are underlying reasons to carry out this type of analysis on urban networks. As mentioned earlier, power law distributions are behind most of the natural networks. This omnipresence is partly due to optimization considerations. Based on thermodynamics reasoning, it is possible to prove that power law based networks are structural optima in terms of energy efficiency and resource consumption. According to Salat and Bourdic [20,21], in many cases, a scaling network is the most efficient structure to distribute a flow within a structure: minimize frictions and energy losses, minimize material consumption and maximize accessibility.

Applied to urban networks, the potential of fractal analysis to improve the structural efficiency is tremendous. The street width/length analysis is one example among others, but this analysis can be extended to most of the urban networks (energy, transport, water, heat, information...). According to Kühnert et al. [22], some urban supply systems follow scaling laws. Mature public transport networks also tend to scale. Based on a simple analysis, Paris Métropolitain or the London tube proves to be implicitly structured by a power law. The number of connexions in a station and the number of stations scrupulously respect a power law with a scaling parameter equal to 0.33 in Paris and 0.35 in London. The volume of passengers in London tube stations also scales, with a scaling parameter equal to 0.5 .

The emergence of power law distributions both in natural phenomena and manmade complex systems is no accident, but the result of a hidden structural optimization. A wide range of questions still remain though. What is the impact in terms of efficiency of urban networks' structural distributions? This issue 
sounds critical in the current context of race to energy efficiency. What is the role of the scaling parameter in terms of urban efficiency and resilience? Bourdic and Salat [23] shed some light on this point by providing a fractal interpretation to Newman and Kenworthy's well known curve that links urban density to energy consumption per capita for private transport. The potential of this type of approach is noteworthy. But one of the prerequisites to reach full potential is a more systematic approach of urban complexity, based on quantitative tools assessing urban systems' hierarchy and its impact on urban efficiency and resilience.

\section{Urban structures and textures}

Another example of the interest of such a systematic fractal approach of urban structures deals with the urban fabric. As urban morphologists, authors base their reasoning on a structural and multi-scale understanding of the urban fabric. The buildings, the courtyards and the street define a physical interface - or membrane - between the city and the outside. The very structure of this membrane impacts widely on urban comfort, ventilation, pollutant dispersion, light availability, heating and cooling. Numerous Euclidian parameters already exist to describe the morphology of this interface and its influence: porosity, sky view factor, the volume to envelope ratio, the passive volume ratio, etc... But, once again, significant results can be obtained by systematizing fractal methods and analysis. Switching from urban networks to the urban fabric theoretically boils down to adding one dimension to the problem. The organisation of empty and filled spaces (buildings, courtyards and streets) is about nothing but optimizing a complex interface between the inside, public spaces and the outside. The sequence of streets and courtyards impacts on the natural cooling through the well known Venturi effect, impacting at the same time on the public space comfort, on cooling and heating requirements. The structure of the membrane impacts on the penetration of light into the urban fabric, impacting at the same time on the lighting requirements.

Optimizing these different parameters on the building scale is nothing but obvious and requires advanced models. Yet switching from the building scale to the neighbourhood, district or city scale is even more difficult. In theory, there is no reason for a sum of optimal buildings to lead to an optimal urban fabric on the district or city scale. The more one scales up, the more interactions appear within the urban fabric. In the end, following West and Salingaros' theoretical work [24], the urban fabric is the superimposition of short, medium and long range interactions. Shading effects appear when one scales up from one to two buildings, then aerolic effects on the neighbourhood scale, then urban heat island effects on the district and city scales. Complexity tremendously increases when scaling up from the building to the city scale, jeopardizing at the same time the potential of bottom-up aggregative methods.

Optimizing the urban membrane on the city scale is thus a complex multiconstraint optimization, depending on a wide range of parameters (degree hour, light, wind, insulation...). As often, the overall optimization corresponds to local 
(both by sector and spatially) sub optima instead of optima. Once again, authors argue that fractal and power law based methods may help for this complex multiparameter optimization. The following example shows how the scale hierarchy of the urban fabric (buildings and courtyards) impacts on the passive volume ratio. The concept of passive zone is described in the LT-method [25] as being the area in the building within a distance from a perimeter wall, usually between 6 and 8 meters. These passive zones benefit from natural lighting and natural ventilation, but also from useful solar gains in winter. The energy consumption associated with lighting and ventilation is thus expected to be lower in these zones, an important part of lighting and ventilation being 'free'.

The following analysis is based on the neighbourhood scale. It aims at showing how passive volume ratio may increase as urban fabric becomes more complex. Figure 4 displays three existing urban morphologies compared with theoretical analogue structures. The complexity of the urban fabric - the scale hierarchy - increases from the left to the right.

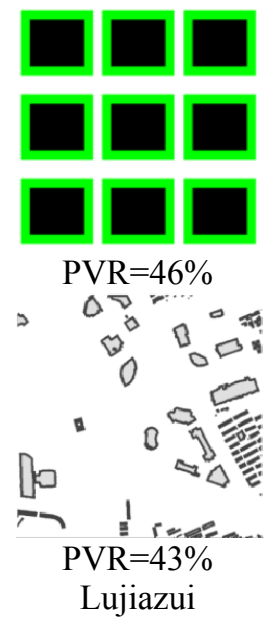

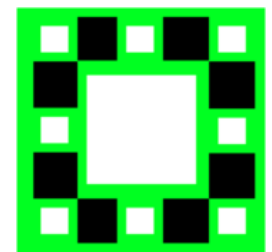

$\mathrm{PVR}=60 \%$

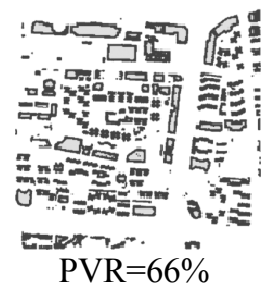

Tianhe district

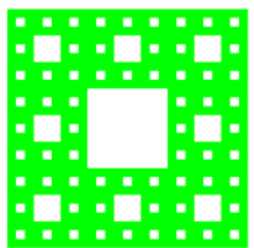

$\mathrm{PVR}=100 \%$

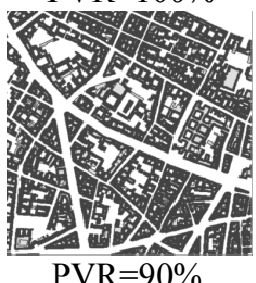

Parisian district

Figure 4: $\quad$ Passive volume ratios (PVR) for 3 theoretical (top, PV in green) and 3 existing (bottom, PV in dark grey) urban morphologies. (See online for colour version.)

This simple geometric analysis shows that complex urban fabrics display a much higher passive volume ratio than simple ones. Fractal theory is a way to optimize the "urban membrane" - the interface between the inside and the outside. The careful reader will then certainly notice that the multiplicity-size distribution of courtyards in the two structures on the right hand side follows a power law. Further research is currently carried out to understand how size and scale hierarchy of courtyards impact on energy consumption patterns according to the different climates. Pushing further this geometric analysis, authors have investigated numerous urban tissues, historical and modernist ones, in cold and 
hot climates. When analyzing real cities, the same kind of results emerges: the more scale hierarchic the urban tissue, the higher the passive volume ratio.

\section{Conclusions}

The major problem of the contemporary city is the disconnection between scales. The $20^{\text {th }}$ century technicist urban planners who ignored the fractal structure of historical cities divided the city into two spatial scales dedicated to two types of relations and behaviours: the greater metropolitan region traversed and structured by large transit infrastructures dedicated to speed and summarily zoned; and the neighbourhood, celebrated as the building block of the sustainable city, when its concept, boundaries and limits remained blurry and ill-defined. The stance adopted as a result involved razing the old fabric and inordinately enlarging the urban grid to bring it in line with the major regional throughways. We know today that this approach is a failure, that it engenders inhuman cities, entirely given over to speed and to the ever-growing intensification of transports and energy consumption. The reductionist approach associated with modernism has not only leaded to a dehumanization of cities. It has also leaded to structurally inefficient urban tissues. Modernist planning has been unable so far to grasp the complexity of historical urban structures that make them be climaxes of efficiency, of interaction between people and of value creation.

Fractal theory provides extremely beneficial insights to better understand the city, and the contributions of Salingaros, Batty, Longley or Frankhauser have been critical. However, there is a need for pushing the analysis further than a city or building rank/size one, or a boundary/area one. Authors' understanding is that a move towards a systematic multi-scale fractal understanding of urban entities and urban facts could be extremely beneficial. Fractal approaches are one of the seldom frameworks able to encompass the complex and multi-scale characteristics of cities. Regarding energy aspects, it is susceptible to provide critical insights on the structural efficiency and resilience of cities (see [20]). Scaling laws are the result of complex multi-scale optimizations both in natural systems and mature large scale man-made ones. Understanding the role of scaling laws in the urban world could help making cities more efficient and sustainable, by speeding up this "maturation" process. But to do so, there is an urgent need for tools allowing grasping the inherent complexity of urban structures. Simple and adapted but robust, quantitative and science-based tools fostering the use of scaling laws to structure cities, and that can be used by every stakeholder of the urban value creation chain.

Beyond urban network and the urban fabric, the framework and scheme authors propose aims at being extended towards socio-economic issues. By analogy, socio-economic tissues may benefit from a more systemic understanding, based on fractal approaches. Building on the seminal work carried out by Alexander [8] and Alexander et al. [26] and then carried on by Salingaros and West [24] and Salingaros [27], investigating the role of scale hierarchy in urban economics may lead to interesting findings, keeping in mind two systematic questions: what makes a city living, and what makes a city 
profitable and creative. Mangin and Panerai's thought [28] that links the value creation in Manhattan throughout the $20^{\text {th }}$ century to extraordinary complexification ability is outstanding. In New York or Paris for instance, the grid has been thought out firstly to define urban subdivisions before defining a transport network. Such a socio-economic analysis may first focus on the impact of urban subdivision structure on land value and economic value creation.

\section{References}

[1] Salingaros, N., Principles of Urban Structure. Techne Press, Neth., 2005.

[2] Mandelbrot, B., The fractal geometry of nature. SF: Freeman \& Co, 1982.

[3] Batty, M. \& Longley, P., Fractal Cities: a Geometry of Form and Function. Academic Press, 1994.

[4] Frankhauser, P., La fractalité des structures urbaines. Paris: Anthropos, 1994.

[5] Pareto, V., Cours d'Economie Politique. Geneva: Droz, 1896.

[6] Zipf, G.K., Human Behaviour and the Principle of Least Effort. A-W, 1949.

[7] Mandelbrot, B., "The variation of certain speculative prices," J. Bus. 36, pp. 394-419, 1963.

[8] Alexander, C., "A city is not a tree," Design, vol. 206, pp. 46-55, 1965.

[9] Schumpeter, J., "Vilfredo Pareto (1848-1923)," Quant. J. Econ. 63, pp. 147-172, 1949.

[10] Batty, M., Hierarchy in Cities and City Systems, CASA Working Papers, Ed.: UCL, 2004.

[11] Pumain, D., "Settlement systems in the evolution," Geo. An. 82B, pp. 73$87,2000$.

[12] Batty, M., "The Size, Scale, and Shape of Cities," Science, Vol 319, pp. 769-71, 2008.

[13] Gabaix, X., "Power laws in economics and finance," An. Rev. Econ. 1, pp. 255-93, 2009.

[14] Champernowne, D., "A model of income distribution," Econ. J. 83, pp. 318-51, 1953.

[15] Simon, H., "On a class of skew distribution functions," Biom. 44, pp. $425-$ 440, 1955.

[16] Salat, S., Cities and Forms, On Sustainable Urbanism.: Hermann, 2011.

[17] Chen, Y., "A Wave-Spectrum Analysis of Urban Population Density: Entropy, Fractal, and Spatial Localization," DDNS, 2008.

[18] Suzuki, H., Dastur, A., Moffatt, S., Yabuki, N., \& Maruyama, H., "Eco ${ }^{2}$ Cities: Ecological Cities as Economic Cities," World Bank Training series, 2010.

[19] Le Corbusier, "Ville contemporaine de trois millions d'habitants", 1922.

[20] Salat, S. \& Bourdic, L., "Scale Hierarchy, Exergy Maximisation and Urban Efficiency", ELCAS2, 2011.

[21] Salat, S. \& Bourdic, L., "Power laws for energy efficient and resilient cities," Proc. Eng., 2011. 
[22] Kühnert, C., Helbing, D. \& West, G.B., "Scaling laws in urban supply networks," Physica A 363, pp. 96-103, 2006.

[23] Bourdic, L. \& Salat, S., "Urban density and private transport energy consumption: A fractal model," in 16th HKSTS, 2011.

[24] Salingaros, N. \& West, B.J., "A universal rule for the distribution of sizes," Env. Plan. B: Plan. \& Des., vol. 26, pp. 909-23, 1999.

[25] Baker, N. \& Steemers, K., "LT Method 3.0 - a strategic energy-design tool for Southern Europe," Energy and Buildings 23, pp. 251-256, 1996.

[26] Alexander, C., Neis, H. \& Anninou, A., A New Theory of Urban Design. New York, Oxford University Press, 1987.

[27] Salingaros, N., A Theory of Architecture. Umbau-Verlag, Germany, 2006.

[28] Mangin, D. \& Panerai, P., Projet Urbain, Eupalinos, Ed.: Parenthèses, 1999. 